\title{
A Comparison of a Luminescence-Based VPSE and an Electron-Based GSED for SE and CL Imaging in Variable Pressure SEM with Conventional SE Imaging.
}

\author{
B. J. Griffin ${ }^{1}$, D.C. Joy ${ }^{2}$ and J. R. Michael ${ }^{3}$
}

1 Centre for Microscopy, Characterization and Analysis, The University of Western Australia, Crawley, WA Australia 6009

2 Center for NanoPhase Materials Science, Oak Ridge National Laboratory, Oak Ridge, TN 37831

3 Sandia National Laboratories, PO Box 5800, Albuquerque, NM 87185-0886

Two detector strategies are used to image the secondary electron (SE) signal in variable pressure and environmental scanning electron microscopy (VP/ESEM). The longest-standing approach is to measure the current in a positively biased detector [1]. This style of detector has various forms that include on-axis concentric plates, grids and even needles or off-axis plates. Collectively these various forms are here collectively termed gaseous secondary electron detectors (GSED). A parallel approach uses a similar biased element but images with the specimen current that is generated through the electron cascade amplification [2]. The second method is to collect the luminescence of the gas ions as they recombine at or near a positively biased element near the detector light guide. The gas ions are generated by an electron cascade amplification process identical to that involved in the GSED operation. The luminescence-based detector is here termed the VPSED. Conventional high vacuum SE imaging employs the well-known Everhart-Thornley SED (ET-SED) and more recently the In-Lens (IN-SED) variants.

This work is the first direct investigation to carefully compare the SE images resultant from GSED or VPSED operation. The project stems from previous and current work where the information content of SE images, under identical collection conditions, has been shown to vary widely [3].

The first experiment has been to image a coated polished copper standard sample. This sample has been imaged at $15 \mathrm{kV}$ and low beam current conditions with IN-SED and ET-SED from most current microscope models. The VPSED and GSED imaged are comparable. The images lack the high contrast of the surface contaminants on the copper shown by high vacuum IN-SED and to a lesser extent some ET-SED (figure 1). Results from specimen biasing experiments will be used to suggest that all of the variable pressure images are BSE-rich.

The data is considered to prove that under most conditions the samples imaged by GSED or VPSED in VP mode charge positive and so trap the bulk of the low energy secondary electron signal.

The second experiment has been to image a strongly cathodoluminescent sample, comprised of polished zircon mineral grains using GSED and VPSED. The VPSED has been found to provide excellent CL images when the biased element of the detector is at zero or negative potential. As the bias becomes increasingly positive, the CL contrast decreases and is lost under normal VPSED operating biases. The GSED shows no CL response, as expected. However the contrast previously described as "Charge Contrast Imaging [4] (CCI)" becomes visible at higher GSED biases for low chamber gas pressures. The CCI contrast is inverse WRT to the CL image. In the case of the VPSED the CCI contrast is suggested to progressively cancel the CL contrast with increasing detector element bias (figure 2). 
The data indicates that the VPSED is well suited to the imaging of CL-emitting materials and for these materials CCI capability is severely compromised. The GSED is best suited to CCI.

Sample biasing has also been investigated under variable pressure conditions. The GSED images show a strong increase in signal-to-noise $(\mathrm{S} / \mathrm{N})$ when the stage is negatively biased under conditions where the total sample-detector bias is constant. It has been observed that negative stage biasing also increases the maximum potential that can be achieved between sample and detector with a consequent increase in $\mathrm{S} / \mathrm{N}$. An improvement up to a factor of $\mathrm{x} 2$ in the $\mathrm{S} / \mathrm{N}$ has been measured. No significant change is observed in VPSED imaging with negative stage bias.

Negative stage biasing up to $100 \mathrm{v}$ provides significant improvements in imaging with GSED. The improvement is considered to be due to a combination of the increased potential between sample and detector, and reduction in signal losses due to attraction of ions to the negatively charged stage. The latter effect is considered similar to the operation of the previously reported "widget" grid [5].

\section{References}

(1) Danilatos, G. C. (1990) Adv. Electronics Electron Phys. 71, 1-102.

(2) Farley, A. N. and Shah, J. S. (1988) Inst. Phys. Conf. Ser. 93, 241-242.

(3) Griffin, B.J., Joy, D.C. and Michael, J.R. (2009) Microsc Microanal, 15 (S2:Proc.), 46-7.

(4) Griffin, B. J. (2000) Scanning 22, 234-242.

(5) Craven, J. P., Baker, F. S., Thiel, B. L., and Donald, A. M. (2002) J. Microsc. 205, 96-105.

(6) Lauren Fisher, is acknowledged for her untiring efforts with data collection.

(7) Support of the UWA Office of Industry and Innovation is also acknowledged.
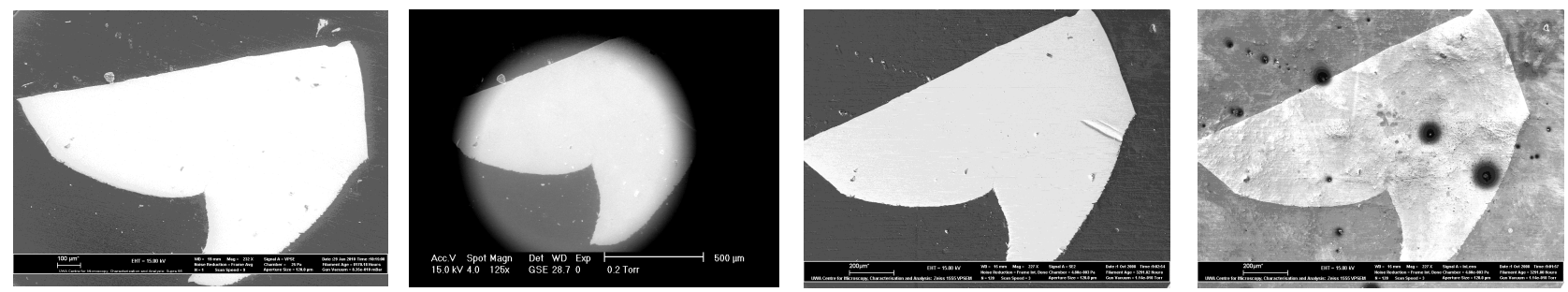

Figure 1: VPSED, GSED, ET-SED and IN-SED images ( $l$ to $r$ ) of a C-coated polished Cu standard embedded in epoxy resin. $E_{0}=15 \mathrm{kV}$. Surface contamination and charging are visible in the INL SEI.
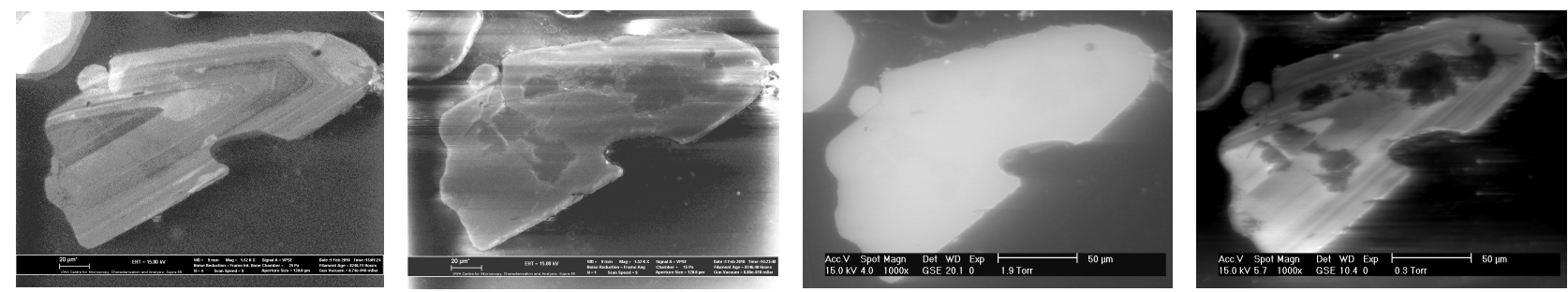

Figure 2: Negatively (CL-rich) to positively (SE-rich) biased VPSED and high gas pressure (SE-rich) to low gas pressure (CCI) GSED images ( $l$ to $r$ ). Both CL and CCI images show the primary growth structure of the zircon but the contrast is inversed. 